ORIGINAL ARTICLE

\title{
Lung cancer cell migration is regulated via repressing growth factor PTN/RPTP $\beta / \zeta$ signaling by menin
}

\author{
Z-J Feng ${ }^{1}, \mathrm{~S}-\mathrm{B} \mathrm{Gao}^{1}$, Y Wu${ }^{1}, \mathrm{X}-\mathrm{F} \mathrm{Xu}{ }^{2}, \mathrm{X} \mathrm{Hua}^{3}$ and G-H Jin ${ }^{1}$ \\ ${ }^{1}$ Department of Basic Medical Sciences, Medical College, Xiamen University, Xiamen, Fujian, PR China; ${ }^{2}$ Department of Pathology, \\ Xiamen Second Hospital, Xiamen, Fujian, PR China and ${ }^{3}$ Department of Cancer Biology, University of Pennsylvania, Philadelphia, \\ $P A, U S A$
}

\begin{abstract}
Menin encoded by the multiple endocrine neoplasia type 1 (MEN1) gene is associated with chromatin and the nuclear matrix and exerts multiple biological functions including regulation of cell proliferation and adhesion. Men1 mutations increase the likelihood of lung cancer development in mice. Menin expression is reduced in certain human non-small cell lung cancer cells, and reduction of menin is closely correlated with increased lung cancer metastasis to lymph nodes. However, it is poorly understood whether menin affects migration of lung cancer cells. In this study, we show that meninregulated A549 lung cancer cell migration, which was mediated by growth factor pleiotrophin (PTN) and its cell surface receptor, protein tyrosine phosphatase beta/zeta (RPTP $\beta / \zeta)$. Ectopic menin expression significantly repressed PTN transcription, but indirectly inhibited RPTP $\beta / \zeta$ expression through repressing PTN expression. Further studies revealed that menin-regulated cell migration through PTN/RPTP $\beta / \zeta$, in conjunction with integrin $\alpha_{v} \beta_{3}$, focal adhesion kinase, phosphatidylinositol 3-kinase and phosphorylated extracellular signal regulated kinase 1/2. These findings provide mechanistic insights into the molecular basis for menin/PTN-mediated regulation of A549 lung cancer cell migration.

Oncogene (2010) 29, 5416-5426; doi:10.1038/onc.2010.282; published online 19 July 2010
\end{abstract}

Keywords: lung cancer; migration; Men1; pleiotrophin; $\operatorname{RPTP} \beta / \zeta$

\section{Introduction}

Menin encoded by the multiple endocrine neoplasia type 1 (MEN1) gene is a nuclear protein, which is mutated in patients with an inherited tumor syndrome, MEN1 (Chandrasekharappa et al., 1997). In endocrine tumors with a germline mutation in one of the $M E N 1$ alleles, the remaining wild-type $M E N 1$ allele is often inactivated because of a somatic mutation (loss of heterozygocity),

Correspondence: Dr G-H Jin, Department of Basic Medical Sciences, Xiamen University, Daxue Road 168, Xiamen 361005, PR China.

E-mail: ghjin@xmu.edu.cn or Dr X Hua, Department of Cancer Biology, University of Pennsylvania, Philadelphia, PA, USA.

E-mail: huax@mail.med.upenn.edu

Received 11 January 2010; revised 27 May 2010; accepted 31 May 2010; published online 19 July 2010 indicating $M E N 1$ as a bona fide tumor suppressor gene in endocrine tumors (Lemos and Thakker, 2008). As menin does not show an obvious homology to any known protein motifs, it has been challenging to elucidate how menin acts as a tumor suppressor.

Recently, multiple lines of evidence suggest that menin is associated with chromatin and nuclear matrix and exerts multiple biological functions including regulation of cell proliferation, apoptosis and DNA repair (Gao et al., 2008). These diverse menin functions may be largely attributed to the crucial role of menin as a scaffold protein, which can alter histone tail modifications and epigenetic status of its target genes to control their transcription (Wu and Hua, 2008). For instance, menin represses proliferation of pancreatic islet cells and mouse embryonic fibroblasts (MEFs) partially through upregulating transcription of $\mathrm{p} 18^{\text {Ink } 4 c}(\mathrm{p} 18)$ and $\mathrm{p} 27^{\text {Kip } 1}$ (p27), potent inhibitors of cyclin-dependent kinases (Schnepp et al., 2006). In this regard, menin interacts with mixed lineage leukemia proteins (Karnik et al., 2005; Milne et al., 2005), histone H3 methyltransferases that catalyze histone $\mathrm{H} 3$ lysine 4 methylation (H3K4me3) with their highly conserved SET domain (Milne et al., 2002). Menin and mixed lineage leukemia bind the $p 18$ and $p 27$ gene loci, enhancing H3K4me3 at these loci, to promote their transcription in endocrine cells, leading to repression of cell proliferation (Karnik et al., 2005; Milne et al., 2005).

Nonetheless, it is still poorly understood whether menin influences development of non-endocrine tumors, such as lung cancer. Lung carcinoids occur sporadically and are also detectable infrequently in patients with MEN1 syndrome (Debelenko et al., 1997, 2000; Petzmann et al., 2001). Non-small cell lung cancers develop in both $\mathrm{MenI}^{+}$

and $p 18^{-/-} ;$Men $^{+/-}$mice, but with a much higher penetrance in mice with the latter genotype (Pei et al., 2007). Further study indicated that $p 18^{-/-}$or $M e n 1^{+/-}$ increased $\mathrm{pRb}$ phosphorylation at cyclin-dependent kinase 4/6 site $\mathrm{Ser}^{608}$, and more obviously, increased $\mathrm{pRb}$ was detected in tumor cells from $\mathrm{pl}^{-1-} ; \mathrm{Men1} \mathrm{I}^{+/-}$mice (Pei et al., 2007). These observations suggest a role for menin in suppressing lung carcinogenesis through regulating cell proliferation. Consistent with this notion, we recently found that menin inhibits proliferation of several human lung cancer cell lines and growth of lung cancer xenograft (Gao et al., 2009). Consistent with a role of menin in suppressing development of lung cancer, in $23 \%$ of 
primary human lung adenocarcinomas, menin expression is significantly reduced. Moreover, a reduction in menin expression is also closely correlated with lymph node metastasis of the cancer (Gao et al., 2009). As cancer metastasis involves cell migration and invasion, these findings raise the possibility that menin may affect cell migration.

Our previous work suggests that menin-mediated suppression of lung cancer is partly through polycomb gene $(\mathrm{PcG})$-dependent repression of pleiotrophin (PTN) expression. PTN is a heparin-binding growth factor that is highly expressed in certain solid cancers, such as in breast and lung cancers (Jager et al., 2002; Perez-Pinera et al., 2007). PTN activates its cell surface receptors, regulating multiple functions including cell adhesion, cell migration, cell proliferation and cytoskeletal stability (Lu et al., 2005; Pariser et al., 2005; Duces et al., 2008). These studies raise the possibility that downregulated menin expression facilitates the development of lung cancer not only by repressing lung cancer cell proliferation, but also by altering lung cancer cell migration and metastasis. However, the precise mechanisms whereby menin regulates cell migration are not well understood. We have previously shown that menin interacts with a scaffold protein, IQ motif containing GTPase activating protein 1, which links the cytoskeleton to cell adhesion and migration in endocrine cells (Yan et al., 2009). However, it remains poorly understood as to how menin influences migration of non-endocrine tumors, such as lung cancer cells. In this study, we show that menin regulates A549 lung cancer cell migration through downregulating expression of PTN and its cell surface receptor, protein tyrosine phosphatase beta/zeta (RPTP $\beta / \zeta$ ) (Perez-Pinera et al., 2007). PTN-RPTP $\beta / \zeta$ expression and activation of focal adhesion kinase (FAK) and phosphoinositide 3-kinase (PI3K) were repressed by menin, and menin-repressed cell migration involved inhibition of PI3K-extracellular signal regulated kinase (ERK) $1 / 2$ signaling, which is crucial for cell migration. Together, these results suggest a novel menin-PTN pathway that controls cell migration in A549 cells.

\section{Results}

\section{Menin inhibits cell migration and represses PTN/RPTP} $\beta / \zeta$ expression

Previously, we showed that menin expression was reduced in lung adenocarcinomas, and menin reduction was highly correlated with increased lymph node metastasis (Gao et al., 2009). As cancer metastasis usually involves enhanced cell migration, we sought to determine whether one of the functions of menin is involved in controlling cell migration. To this end, we performed a modified transwell chamber assay to evaluate the effect of loss of menin on migration of Men $1^{+1+}$ and $M e n 1^{-1-}$ MEFs. The results indicate that Menl ablation significantly increased cell migration (Figure 1a, $P<0.05$; Supplementary Figure 1a). Next, we examined whether MEN1 knockdown using short hairpin RNAs (shRNAs) affects migration of A549 lung adenocarcinoma cells. Control vector and three distinct shRNAs that specifically target human MEN1 were stably transfected into A549 cells. The resulting cells were analyzed for knockdown of endogenous $M E N 1$ using both quantitative reverse transcriptase-PCR and western blotting. MEN1 shRNAs 2-3 substantially reduced expression of $M E N 1 \mathrm{mRNA}$ and protein, but shRNA1 failed to knockdown MEN1 expression (Figure 1b; Supplementary Figure 1b). Interestingly, correlated with the levels of MEN1 knockdown by shRNAs, shRNA 2-3 significantly increased A549 cell migration $(P<0.05$, respectively), but shRNA1 that was unable to reduce $M E N 1$ expression failed to do so (Figure 1c). Together, these results suggest that menin normally restricts cell migration.

Our previous work has shown that menin suppresses lung cancer cell proliferation partly through epigenetically repressing transcription of growth factor PTN (Gao et al., 2009). PTN is highly expressed in certain solid cancers (Jager et al., 2002; Perez-Pinera et al., 2007), binds to cell surface receptors, such as RPTP $\beta / \zeta$, and has a crucial role in cell proliferation, adhesion and migration ( $\mathrm{Lu}$ et al., 2005; Pariser et al., 2005; Duces et al., 2008). To examine whether menin represses lung cancer cell migration partly through affecting RPTP $\beta / \zeta$ expression, we evaluated the effect of loss of menin on RPTP $\beta / \zeta$ expression in Men1 ${ }^{+/+}$and MenI $^{-/-}$MEFs. Menl excision (Supplementary Figure 1c) increased mRNA expression of RPTP $\beta / \zeta$ (6.5-fold), as detected by quantitative reverse transcriptase-PCR (Figure 1d) and the downregulated PTN mRNA expression (eightfold) by menin has been reported early (Gao et al., 2009). Similar results were also obtained by detection of PTN and RPTP $\beta / \zeta$ using regular reverse transcriptase-PCR (Supplementary Figure 1d). Increased PTN and RPTP $\beta / \zeta$ protein levels were detected in asynchronous Menl null MEFs, as compared with the Men1-expressing cells, using western blotting (Figure 1e). We further determined PTN and RPTP $\beta / \zeta$ expression in the $\mathrm{Men1}^{+/+}$and $\mathrm{Men1^{-/- }}$ MEFs in an exponential growth phase, using immunofluorescence staining. As illustrated in Supplementary Figure 2, the Men1-null cells failed to show menin staining (Supplementary Figures $2 b$ and $\mathrm{j}$ ), but showed an obvious cytoplasmic staining for PTN and RPTP $\beta / \zeta$ (Supplementary Figures $2 \mathrm{c}$ and $\mathrm{k}$ ). Conversely, menin-expressing MEFs showed a clear staining for nuclear menin (Supplementary Figures $2 \mathrm{f}$ and $n$ ), but a reduction of PTN and RPTP $\beta / \zeta$ staining (Supplementary Figures $2 \mathrm{~g}$ and $\mathrm{o}$ ). Together, these results indicate a pivotal role for menin in downregulating both PTN and its receptor, RPTP $\beta / \zeta$ expression. To further confirm these results, we generated three independent pairs of MEFs to analyze the intrinsic biological effect of menin on cell phenotype and target gene expression. Similar results from one of the three pairs of MEFs were presented in Supplementary Figures $3 \mathrm{a}$ and b.

\section{Menin regulates cell migration partly through PTN and RPTP $\beta / \zeta$ signaling}

Next, we examined whether menin regulates PTN and RPTP $\beta / \zeta$ expression in A549 adenocarcinoma cells. 


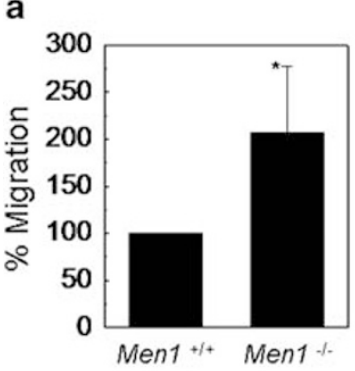

b

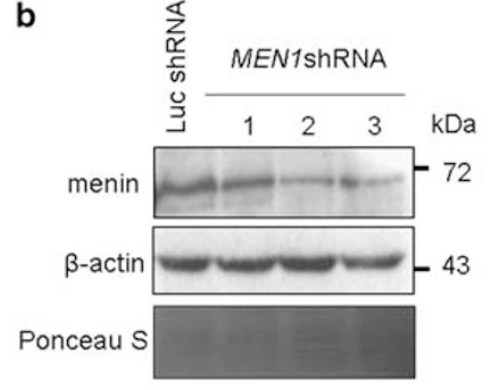

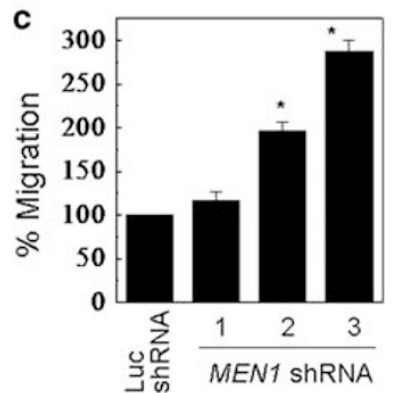

d

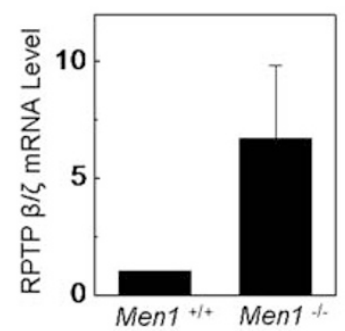

e

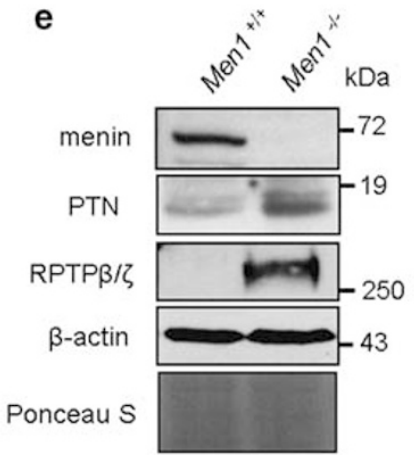

Figure 1 Menin inhibits cell migration and represses PTN/RPTP $\beta / \zeta$ expression. (a) $\mathrm{Men1^{+/+ }}$ and $\mathrm{Men1^{-/- }}$ MEFs were added to the upper-filter of a transwell, and $24 \mathrm{~h}$ later, the migrated cells were stained with crystal violet, and cell number was counted under a microscope. (b) A549 cells were transfected with either vector-expressing shRNAs against Luc or one of the three shRNAs against MEN1 and selected by G418. The efficiency of menin silencing was determined by western blotting. Equal sample loading was confirmed by Ponceau S staining of the Western blot membrane. (c) The selected A549 cells were added to the upper-filter, and cell migration was determined. (d) Increased RPTP $\beta / \zeta$ mRNA levels in Men1 $1^{-1-}$ MEFs were detected by real-time quantitative reverse transcriptase (qRT)-PCR. The amount of RPTP $\beta / \zeta$ mRNA was determined by normalizing their mRNA quantity with the control $\beta$-actin mRNA level, and mean values and s.d. were calculated from triplicates of a representative experiment. (e) The efficiency of menin ablation and the effect of Men 1 expression on PTN $(18 \mathrm{kDa})$ and RPTP $\beta / \zeta(\approx 250 \mathrm{kDa})$ expression were determined by western blotting, and $\beta$-actin was used as a loading control. Equal sample loading was also confirmed by Ponceau $\mathrm{S}$ staining of the western blot membrane. Results in all cases are mean \pm s.d. percentage change in number of migrating cells vs corresponding untreated cells (default $=100$ ) and calculated from triplicates of an independent experiment, ${ }^{*} P<0.05$ vs control.

Our previous work has shown that ectopic menin expression reduces PTN expression (Gao et al., 2009). A549 cells were stably transfected with either control vector or a construct-expressing MEN1 (pMX-menin). Ectopic menin expression was confirmed by western blotting, and as expected, menin overexpression reduced the protein level of RPTP $\beta / \zeta$ (Figure 2a). Further, MEN1 knockdown by shRNA also increased the protein level of RPTP $\beta / \zeta$ in A549 cells (Supplementary Figure 4). We also examined the effect of MEN1 point mutations, A242V and L22R, which were identified from inherited MEN1 patients (Pannett and Thakker, 1999) on RPTP $\beta / \zeta$ expression; we found that $A 242 \mathrm{~V}$ and L22R lost or partially lost the ability to repress RPTP $\beta / \zeta$ expression (Figure $2 b$ ). Correlated with this result, both wild-type menin and L22R mutant overexpression significantly reduced A549 cell migration $(P<0.05$, respectively), however, A242V was unable to reduce RPTP $\beta / \zeta$ expression and did not significantly repress cell migration (Figure 2c). Next, we used an alternative approach, the scratch wound assay, to compare the motility of mock versus MENI-overexpressed A549 cells. The extent of wound closure in control cells within 4 days of wounding was much higher than that in ectopically MEN1-expressed A549 cells
(Figure 2d). The dramatic difference between these two cells reinforces the notion that menin normally represses cell migration. Next, another pair of stable menin overexpressed A549 cell lines was established by using PLNCX2 retrovirus system, and similar results on the role of menin in regulating PTN/RPTP $\beta / \zeta$ expression and cell migration were shown in Supplementary Figures 5a and $\mathrm{b}$. In addition, another human nonsmall cell lung cancer cell line NCI-H157 was used to confirm the above results. It is noteworthy that ectopic menin expression not only inhibited PTN/RPTP $\beta / \zeta$ expression, but also repressed NCI-H157 cell migration (Supplementary Figures $5 \mathrm{c}$ and d). We further tested whether PTN/RPTP $\beta / \zeta$ is required for menin-repressed A549 cell migration. Three distinct RPTP $\beta / \zeta$ shRNAs were transfected into A549 cells, and RPTP $\beta / \zeta$ shRNA3 reduced the RPTP $\beta / \zeta$ protein level effectively (Figure 2e). It is noteworthy that RPTP $\beta / \zeta$ knockdown by shRNA3 reduced migration of A549 cells (Figure 2f). Furthermore, PTN knockdown effectively reduced intracellular PTN expression (Figure 2g), concomitant with reduced A549 cell migration (Figure 2h). Together, these data indicate that menin inhibits A549 cell migration at least partly through repressing expression of PTN and RPTP $\beta / \zeta$. 


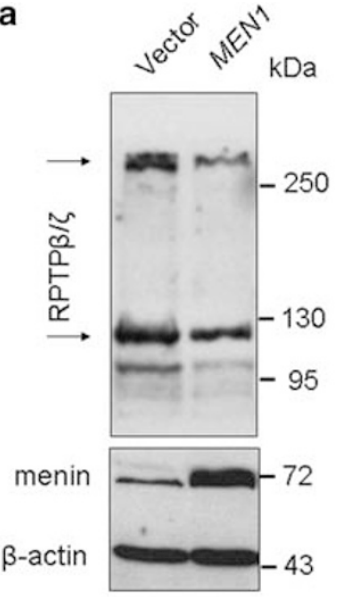

b
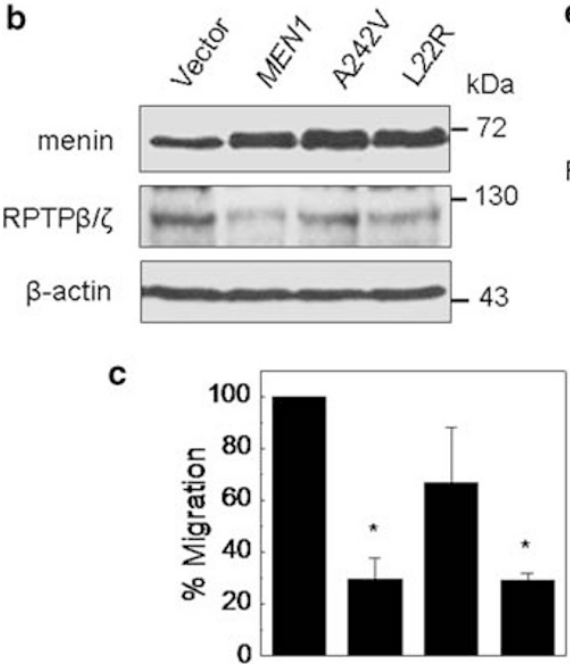

d
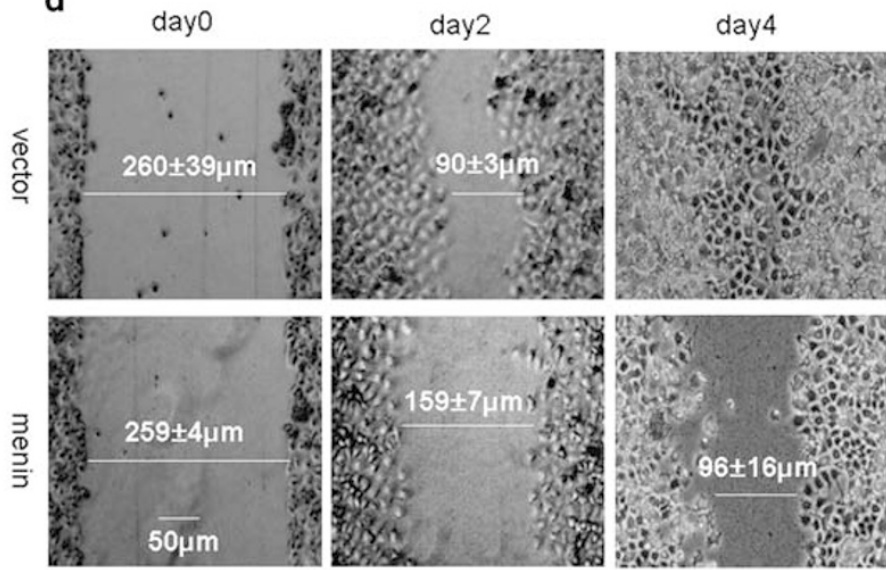

e

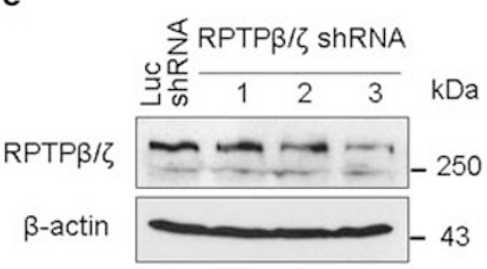

g

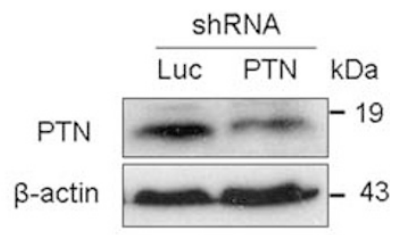

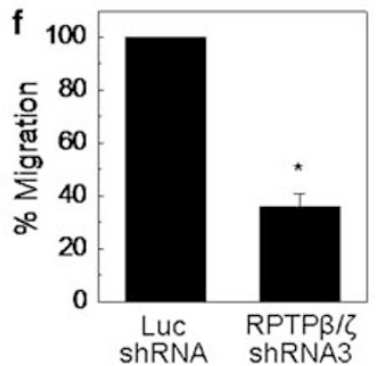

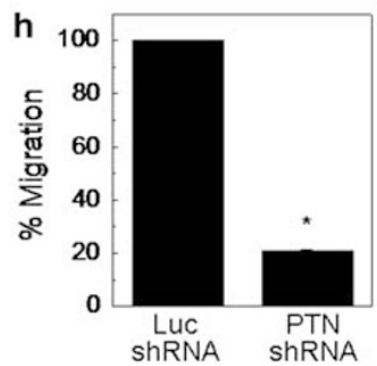

Figure 2 Menin inhibits A549 cell migration partly through repressing PTN and RPTP $\beta / \zeta$ expression. (a) A549 cells were transfected with $M E N 1$ and selected by puromycin. Expression of menin and downregulated RPTP $\beta / \zeta(\approx 250$ and $130 \mathrm{kDa})$ were determined by western blotting. (b) A549 cells were transfected with an empty vector, wild-type MEN1, point mutation A242V or L22R. Menin and RPTP $\beta / \zeta$ expression in the various cell lines above were analyzed by western blotting. (c) The various A549 cell lines were used to determine cell migration. (d) Menin overexpression inhibited closure of artificial wounds made in confluent cellular monolayers. (e) A549 cells were stably transfected with either Luc shRNA or vector expressing one of three distinct RPTP $\beta / \zeta$ shRNAs, and the efficiency of RPTP $\beta / \zeta$ knockdown was determined by western blot. (f) A549 cells transfected with RPTP $\beta / \zeta$ shRNA3 were used to determine cell migration. (g) A549 cells were transfected with PTN shRNA, and the efficiency of PTN knockdown was determined by western blotting. (h) The cell migration of the above A549 cells was determined by three independent experiments, ${ }^{*} P<0.05$.

\section{Menin indirectly regulates $R P T P \beta / \zeta$ expression through PTN}

As menin represses PTN transcription partly through PcG-mediated H3K27me3 at the PTN locus (Gao et al., 2009), we determined if menin also bound to the RPTP $\beta / \zeta$ locus using chromatin immunoprecipitation (IP) assay. However, we failed to detect menin binding to the RPTP $\beta / \zeta$ promoter region (data not shown). This may suggest that menin regulates RPTP $\beta / \zeta$ transcription through an indirect mechanism. PTN is a natural ligand for RPTP $\beta / \zeta$, a phosphatase of phosphorylated tyrosine, and PTN signaling suppresses the phosphatase activity of RPTP $\beta / \zeta$ in reducing steady-state of tyrosine phosphorylation of downstream signaling molecules (Perez-Pinera et al., 2007). Although it is possible that menin represses PTN and RPTP $\beta / \zeta$ separately, we cannot rule out that PTN positively regulates RPTP $\beta / \zeta$ expression.

To further dissect the potential relationship between menin, PTN and RPTP $\beta / \zeta$, we stably transfected A549 cells with PTN shRNA, and the resulting cells were analyzed for the mRNA and protein levels of PTN and RPTP $\beta / \zeta$. Interestingly, PTN knockdown diminished the RPTP $\beta / \zeta$ mRNA and protein levels (Figures 3a and $b$ ). In a complementary approach, we ectopically expressed menin and/or PTN to determine their effect on the RPTP $\beta / \zeta$ mRNA level. As shown in Figure 3c, menin overexpression reduced both the PTN and RPTP $\beta / \zeta$ mRNA levels (lane 2); notably, ectopic PTN expression abrogated menin-induced reduction of the 
a

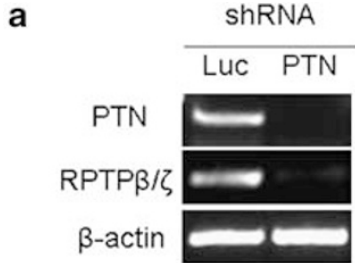

b

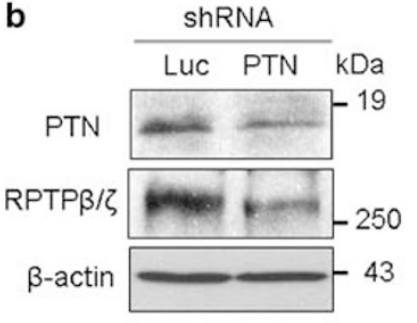

C

\begin{tabular}{|c|c|c|c|}
\hline MEN1 & & + & + \\
\hline PTN & & & + \\
\hline Vector & + & + & \\
\hline Lane & 1 & 2 & 3 \\
\hline MEN1 & & \\
PTN & \\
RPTP $3 / \zeta$ & \\
B-actin & \\
\hline
\end{tabular}

d

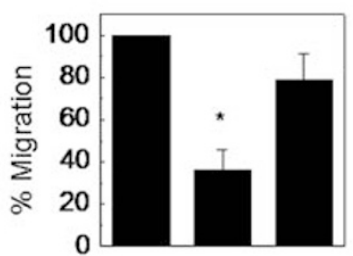

e
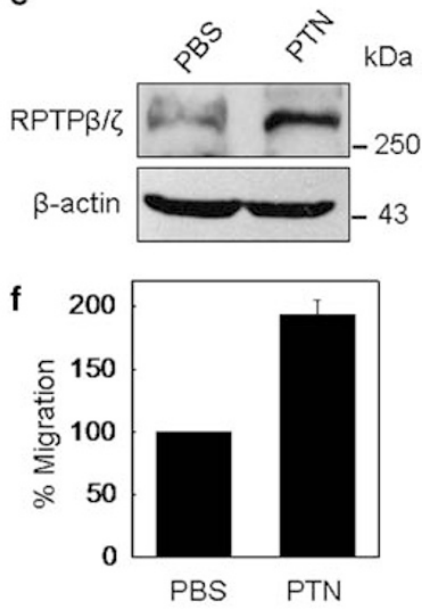

Figure 3 MEN1 indirectly regulates RPTP $\beta / \zeta$ expression through PTN in A549 cells. (a, b) A549 cells were transfected with PTN shRNA, and the resulting cells were processed to determine PTN and RPTP $\beta / \zeta$ mRNA and protein levels using reverse transcriptase (RT)-PCR and western blotting, respectively. (c) A549 cells were transfected with either control vector (30 $\mu \mathrm{g})$ or construct expressing either MEN1 (MEN1 $15 \mu \mathrm{g}+$ vector $15 \mu \mathrm{g})$, or co-transfected with $M E N 1$ and PTN-expressing constructs $(M E N 115 \mu \mathrm{g}+\mathrm{PTN} 15 \mu \mathrm{g})$. The resulting cells were processed to determine menin, PTN and RPTP $\beta / \zeta$ mRNA levels by RT-PCR. (d) The resulting cells were monitored for their migration, $N=3,{ }^{*} P<0.05$ vs control. (e, f) recombinant human PTN (rhPTN) $(100 \mathrm{ng} / \mathrm{ml})$ increased RPTP $\beta / \zeta$ expression and migration of A549 cells.

RPTP $\beta / \zeta$ mRNA level (lane 3). Consistent with this observation, menin overexpression significantly reduced A549 cell migration (Figure $3 \mathrm{~d}, P<0.05$ ), but PTN abrogated menin-induced reduction of cell migration (Figure 3d). Furthermore, addition of recombinant human PTN to culture medium upregulated the RPTP $\beta / \zeta$ protein level, as shown by western blotting (Figure 3e), as well as A549 cell migration (Figure 3f). Together, these findings strongly suggest that PTN upregulates RPTP $\beta / \zeta$ expression, whereas menin represses RPTP $\beta / \zeta$ expression indirectly through inhibiting PTN expression.

\section{PTN increases interaction between RPTP $\beta / \zeta$ and integrins}

Integrin $\alpha_{v} \beta_{3}$ is important for cell signaling, survival and migration particularly during angiogenesis and tumorigenesis (del Pozo et al., 2004). However, the role of integrins for the migration of lung cancer cells has not been analyzed extensively. Integrin $\alpha_{v} \beta_{3}$ is a potential receptor for PTN and is required for PTN-induced endothelial cell migration through RPTP $\beta / \zeta$ (Mikelis et al., 2009). Thus, PTN/RPTP $\beta / \zeta$ signaling may affect lung cancer cell migration in conjunction with integrins. Our results indicate that RPTP $\beta / \zeta$ expression was stimulated by PTN in A549 cells (Figure 3e). It is noteworthy that immunofluorescence staining results indicate that PTN substantially increased expression of both RPTP $\beta / \zeta$ and integrin $\beta 3$ and colocalization of RPTP $\beta / \zeta$ and integrin $\beta_{3}$ in the A549 cells (Figure 4a). This result prompted us to analyze whether $\alpha_{\mathrm{v}} / \beta_{3}$ associates with RPTP $\beta / \zeta$. We performed co-IP from extracts of A549 lung cancer cells and found that endogenous integrin $\alpha_{\mathrm{v}}$ and $\beta_{3}$ were co-immunoprecipitated with endogenous RPTP $\beta / \zeta$ (Figure 4b). As a control, IgG failed to pull down $\alpha_{\mathrm{V}}$ and $\beta_{3}$ (Figure $4 b$ ). The interaction between $\alpha_{v}$ and RPTP $\beta / \zeta$ was further confirmed by IP of endogenous RPTP $\beta / \zeta$ with $\alpha_{v}$ antibody (Figure 4c). In addition, interaction between $\alpha_{v}$ and $\beta_{3}$ was also detected in A549 cells (Figures $4 \mathrm{c}$ and $\mathrm{d}$ ). We further examined the effect of menin on $\alpha_{\mathrm{v}}$ and $\beta_{3}$ expression in A549 cells and in Men $1^{+/+}$and $M e n 1^{-/-}$MEFs. Western blotting revealed that menin overexpression did not affect expression of either $\alpha_{\mathrm{v}}$ or $\beta_{3}$ in A549 cells (Supplementary Figure 6a) and similar results were obtained in Men 1 null MEFs (Supplementary Figure 6b), suggesting that PTN transiently increases the integrin expression and their colocalization with RPTP $\beta / \zeta$. Overall, the results above indicate that PTN stimulation increases expression of both RPTP $\beta / \zeta$ and integrins, as well as their interaction.

\section{Menin inhibits activation of FAK, PI3K and ERK1/2, which were crucial for cell migration}

RPTP $\beta / \zeta$ is an important regulator in the reciprocal control of the steady-state tyrosine phosphorylation levels of $\beta$-catenin by tyrosine kinases and phosphatases (Meng et al., 2000). $\beta$-Catenin acts as a key factor in the E-cadherin-mediated cell-cell adhesion (Nelson and Nusse, 2004). To elucidate cell signaling underlying menin/PTN-regulated cell migration, we tested if the menin-regulated cell migration requires $\beta$-catenin signaling. However, no substantial difference in expression of $\beta$-catenin or its Tyr 142 phosphorylation was detected in both A549 and MEFs (Supplementary Figures 6a and $b$ ), raising the possibility that other key factors link menin/PTN signaling to cell migration. FAK, a protein tyrosine kinase that is recruited at an early stage to focal 
a
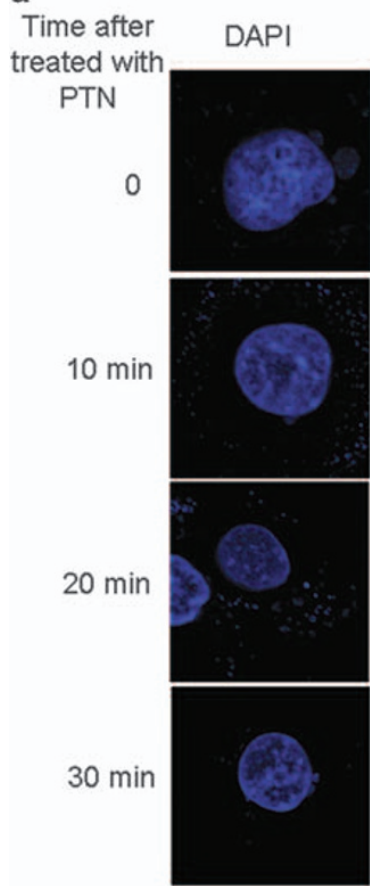
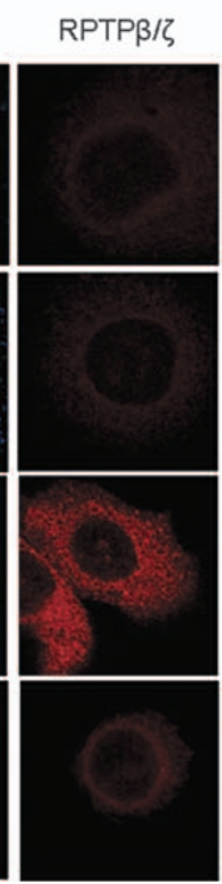
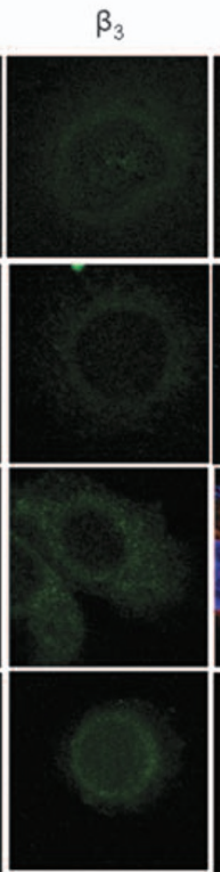
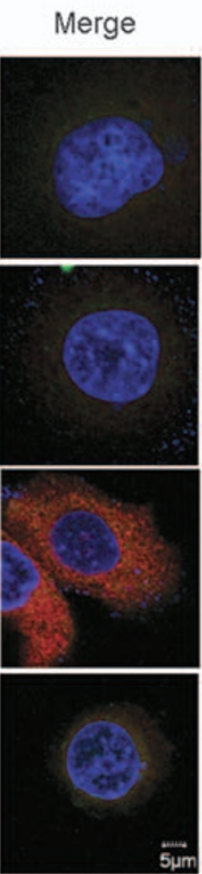

b
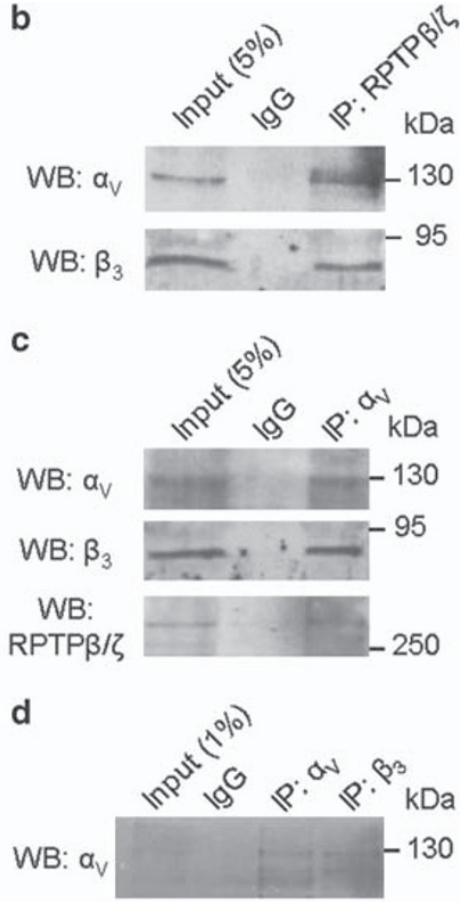

Figure 4 PTN increases interaction of RPTP $\beta / \zeta$ and integrins. (a) Serum-starved A549 cells were treated with $100 \mathrm{ng} / \mathrm{ml}$ recombinant human PTN (rhPTN) and harvested at various time points. RPTP $\beta / \zeta$ (red), $\beta_{3}$ (green) and 4,6-diamidino-2-phenylindole (DAPI) (blue) were detected by IF, $\times 1600$-fold. (b-d) A549 cell lysates were IP for RPTP $\beta / \zeta, \alpha_{v}$ and $\beta_{3}$, and immunoprecipitates were analyzed by western blotting for the presence of RPTP $\beta / \zeta, \alpha_{v}, \beta_{3}$.

adhesions, mediates many downstream adhesion responses, including activation of the p85-subunit of PI3K (Baillat et al., 2008). To examine whether menin affects FAK, we evaluated the effect of loss of menin on expression of FAK in $\mathrm{MenI}^{+/+}$and $\mathrm{Men1}^{-/-}$MEFs. Men 1 excision did not affect the total amount of FAK, but substantially increased the level of its Tyr 397phosphorylated form (Figure 5a). We further determined the effect of menin on PI3K and ERK1/2, other downstream effectors of FAK (Baillat et al., 2008), in the MEFs. The results indicate that Menl excision increased expression and consequently, phosphorylation (Tyr458) of PI3K, as well as ERK1/2 phosphorylation (Thr202/Tyr204) (Figure 5a). Indeed, similar results on menin's role in reducing the phosphorylated forms of FAK and ERK1/2 were obtained in menin overexpressed A549 cells (Figure 5b). Consistently, the menin-null MEFs showed obvious cytoplasmic staining for $p E R K 1 / 2$, while menin-expressing MEFs showed much weaker staining for $p$ ERK1/2 (Supplementary Figure 6c). Collectively, these results suggest that FAK signaling may link menin/PTN to cell migration partly through regulating PI3K and ERK1/2 phosphorylation.

Cell morphology and migration are known to be regulated by members of the Rho family of small GTPases, including Rho, Rac1 and Cdc42 (Hall, 1998). Rac1 and Cdc42 are activated by ligation of integrins, whereas Rho activation relies on either integrins, syndecan-4 or additional cell-surface receptors (Hood and Cheresh, 2002). Hence, we further examined if menin-regulated PTN expression controls cell migration partly through Rho family signaling. Ectopic menin expression did not alter the amount of either activated forms (GTP-bound) or the total amount of Rho, Rac1 and Cdc42 in A549 cells (Figure 5c). Similar results were obtained in Men1-null or Men1-expressing MEFs (Supplementary Figure 7). Interestingly, the Cdc42GTPase and Racl-GTPase activity were transiently induced by PTN at 10-30 min (Figure 5d), similar to the induction of RPTP $\beta / \zeta$ and integrins by PTN (Figure 4a). As menin inhibited expression of PTN, as well as phosphorylation of FAK and ERK1/2, but did not affect Cdc42 and Racl activities, it is likely that menin-mediated repression of PTN leads to relatively long-lasting repression of the FAK-ERK $1 / 2$, but only transient repression of the Rho family of the GTPases in regulating cell migration.

\section{Menin inhibits FAK-ERK activation through PTN/RPTP $\beta / \zeta$ and partly represses migration through inhibiting ERK1/2 phosphorylation \\ It is unclear how menin regulates FAK activation. We examined whether RPTP $\beta / \zeta$ interacts with FAK in A549 cells, but failed to observe interaction between them using co-IP. It has been reported that integrin- FAK interaction may serve as a downstream effector of PTN (Mikelis et al., 2009), and integrin $\alpha_{\mathrm{v}} / \beta_{3}$ directly interacts with RPTP $\alpha$ or RPTP $\beta / \zeta$, which is essential for integrin $\beta_{3}$ activation (von Wichert et al., 2003; Mikelis et al., 2009). FAK is itself regulated by various}



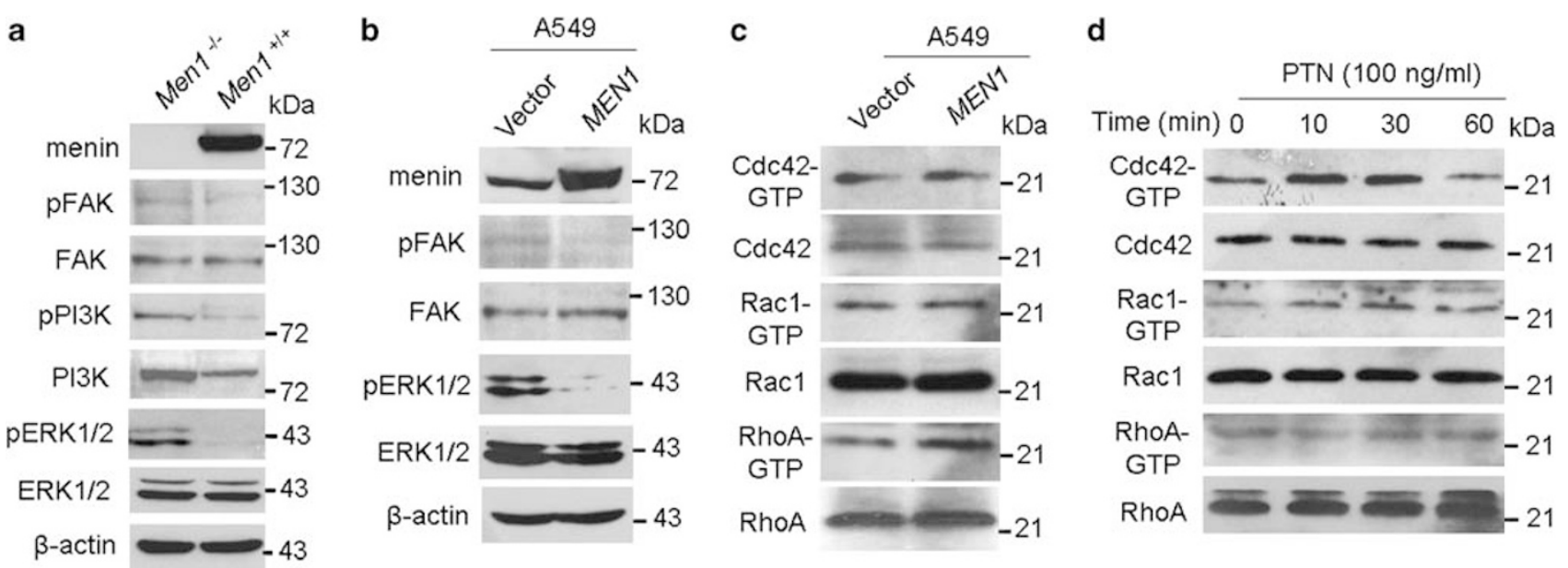

Figure 5 Menin inhibits activation of FAK, PI3K and ERK1/2. (a) The efficiency of menin ablation and its effect on upregulation of pFAK, PI3K ( $85 \mathrm{kDa}$ ), pPI3K and pERK1/2 were determined by western blotting. (b) The effect of ectopic menin expression on FAK (125 kDa), pFAK, ERK1/2 (42 and $44 \mathrm{kDa}$ ) and pERK1/2 in A549 cells were determined using western blotting. (c) PAK1-PBD agarose and Rhotekin RBD agarose were used to isolate GTP-Cdc42, GTP-Racl and GTP-RhoA from whole cell lysates from meninoverexpressing A549 cells. The Cdc42-GTP, Rac1-GTP and RhoA-GTP were detected using western blotting and normalized by the total input protein. (d) Serum-starved A549 cells were treated with $100 \mathrm{ng} / \mathrm{ml}$ recombinant human PTN (rhPTN) and harvested at various time points. The activation of $\mathrm{Cdc} 42$, Racl and RhoA were detected by western blotting and normalized by the total input protein.

mechanisms, including tyrosine phosphorylation, serine/ threonine phosphorylation and protein-protein interactions (McLean et al., 2005). Therefore, we tested if downregulation of FAK phosphorylation is mediated by menin through downregulating PTN/RPTP $\beta / \zeta$ expression. To this end, serum-starved A549 cells were stimulated by addition of recombinant human PTN and allowed to progress for various periods of time before analysis. The results indicate that phosphorylation of FAK was obviously increased after $15 \mathrm{~min}$ of treatment with PTN, reached a peak between 30 and $60 \mathrm{~min}$ after the treatment, but went down after $120 \mathrm{~min}$ (Figure 6a). In contrast, phosphorylation of ERK1/2 was abruptly increased $15 \mathrm{~min}$ after exposure to PTN, but rapidly reduced $30 \mathrm{~min}$ after treatment with PTN (Figure 6a). Consistently, knockdown of PTN or RPTP $\beta / \zeta$ reduced basal level of phosphorylation of FAK and ERK1/2 (Figure 6b). These results suggest that PTN can increase phosphorylation (or activation) of both FAK and ERK1/2; however, as the kinetics of phosphorylation of ERK1/2 and FAK is distinct, they might be activated by the PTN pathway in a distinct manner. Activated FAK has an essential role for maintenance of several cell phenotypes, including cell migration through $\mathrm{PI} 3 \mathrm{~K} /$ mitogen-activated protein kinase signaling (McLean et al., 2005). We thus examined if PI3K and ERK1/2 were involved in menin-regulated cell migration. A549 cells were treated with LY294002 or U0126, respectively, inhibitors for PI3K and MEK $1 / 2$, respectively. The PI3K inhibitor reduced phosphorylation of ERK1/2, but not the total amount of ERK1/2 in A549 cells (Supplementary Figure 8), and decreased A549 cell migration in a dose-dependent manner (Figure 6c). As expected, the migration of A549 cells treated with the MEK1/2 inhibitor was also reduced in a dose-dependent manner (Figure 6c). In addition, we collected six cases with menin downregulated primary lung adenocarcinomas, and found that the levels of RPTP $\beta / \zeta$ and pERK1/ 2 were high, as shown by immunohistochemistry in two of six cases (Supplementary Figure 9). These results suggest that menin-regulated PTN-ERK1/2 pathway could be involved in development of certain lung adenocarcinoma. Together, our results suggest that menin inhibits FAK and ERK1/2 phosphorylation partly through PTN/RPTP $\beta / \zeta$ expression, and ERK1/2 activation may have a role for menin-mediated repression of lung cancer cell migration.

\section{Discussion}

Cell migration is a crucial step of cancer metastasis, a major cause of cancer-related death (Molloy and van 't Veer, 2008). Many oncogenes and tumor suppressor genes are involved in regulating tumor cell migration, invasion and metastasis (Klein, 2008). For example, PTEN is well known for its role in regulating tumor growth, invasion, and metastasis, and it is capable of restricting growth and survival by antagonizing the activity of PI3K (Tamura et al., 1999; Chen et al., 2009a). MEN1, a bona fide tumor suppressor gene in endocrine tumors, exerts multiple biological functions including regulation of cell proliferation (Gao et al., 2008). Until recently, little has been known regarding the function of menin in controlling cancer cell migration.

We found that menin expression in tumors, as compared with the adjacent normal epithelial cells, was markedly reduced or not detectable in 10 out of 45 adenocarcinoma samples, and reduction of menin 

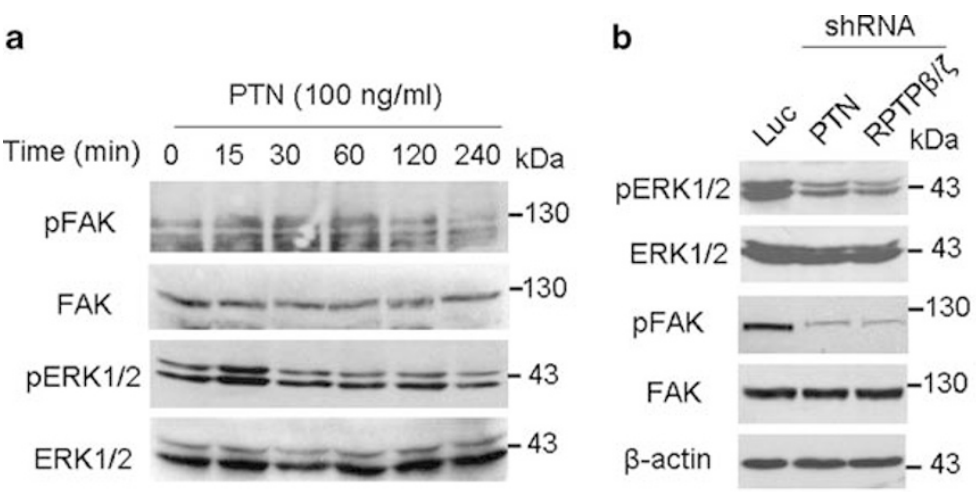

d
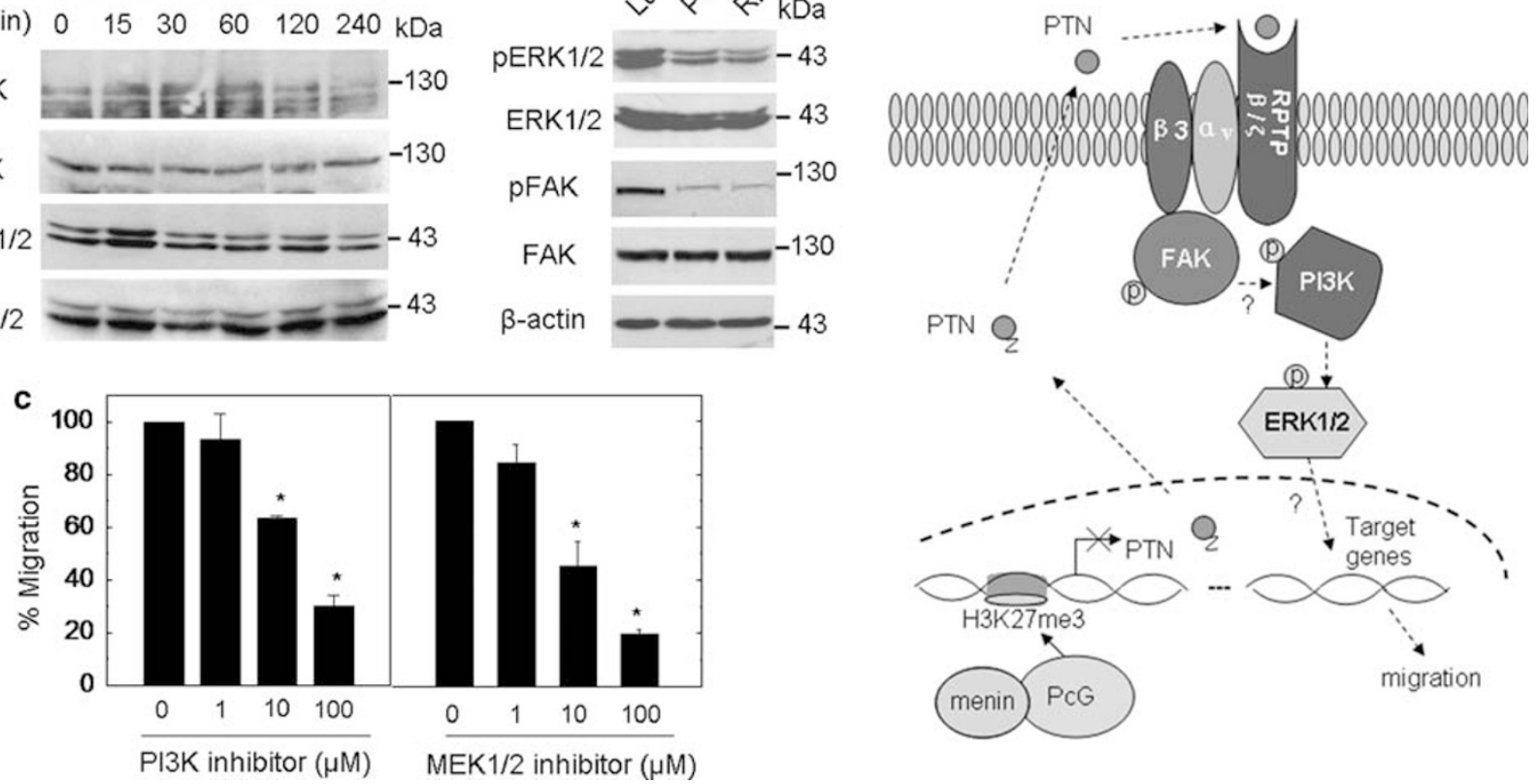

Figure 6 Menin regulates FAK and ERK1/2 activation through PTN/RPTP $\beta / \zeta$ and represses cell migration partly through ERK1/2. (a) Serum-starved A549 cells were treated with $100 \mathrm{ng} / \mathrm{ml}$ recombinant human PTN (rhPTN) and harvested at various time points. The activation of FAK and ERK1/2 were determined with western blotting. (b) A549 cells were transfected with PTN or RPTP $\beta / \zeta$ shRNA, pFAK and pERK1/2 were examined with western blotting. (c) Migration of A549 cells treated with LY294002 or U0126 were significantly reduced in a dose-dependent manner. $N=3, * P<0.05$. (d) A model for regulation of cell migration by menin through growth factor PTN/RPTP $\beta / \zeta$.

expression was correlated with an increase in lymph node metastasis (Gao et al., 2009). Further investigation has revealed that menin represses migration of lung cancer cells, at least partly through inhibiting expression of PTN/RPTP $\beta / \zeta$, a growth factor and its cell surface receptor that are involved in controlling cell proliferation, migration and apoptosis (Perez-Pinera et al., 2007). However, unlike menin-mediated epigenetic repression of PTN transcription through H3K27me3, menin represses RPTP $\beta / \zeta$ expression possibly indirectly through inhibiting PTN expression. It remains unclear how PTN normally upregulates expression of its receptor, RPTP $\beta /$ $\zeta$. As PTN has several candidate receptors including anaplastic lymphoma kinase and integrin $\alpha_{\mathrm{v}} \beta_{3}$ (Stoica et al., 2002; Mikelis et al., 2009), one possibility is that PTN activates these receptors and thus indirectly upregulates expression of RPTP $\beta / \zeta$. Consistent with these scenarios, our previously finding that menin can directly and indirectly downregulate at least two crucial components of the PTN pathway, both PTN and a downstream receptor, anaplastic lymphoma kinase (Gao et al., 2009), respectively, highlights the importance of menin in controlling this signaling pathway.

Integrin signaling is a major pathway in regulating cell adhesion to extra-cellular matrices. Interaction between integrins and their substrates regulates various cellular functions associated with tumor development and metastatic progression, including cell adhesion, migration and invasion (Tamura et al., 1999; Chen et al., 2009 b). Integrin-mediated cell adhesion not only initiates signals directly, but also modulates signaling downstream of growth factor receptors; the integrinFAK signaling pathway enables RhoA to activate its effectors in cell migration (Palazzo et al., 2004). It has been reported that PTN activates signal transduction through RPTP $\beta / \zeta$ or integrin $\left(\alpha_{\mathrm{v}} \beta_{3}\right)$ and migration of endothelial cells (Mikelis et al., 2009). In particular, PTN induces migration of the cells through activating FAK, PI3K and ERK (Polykratis et al., 2005). Our results show that RPTP $\beta / \zeta$ interacted with integrin $\alpha_{v}$ and $\beta_{3}$ in A549 cells, and that menin not only inhibits expression of PTN, but also represses the activation (phosphorylation) of FAK, PI3K and ERK1/2. The FAK signaling complex acts to recruit and/or phosphorylate a number of signaling proteins and is involved in cell adhesion and the motile and invasive phenotype in lung cancer cells (Liu et al., 2008). FAK is upregulated in non-small cell lung cancers (Carelli et al., 2006), and fibronectin-mediated activation of FAK leads to lung cancer metastasis through ERK or PI3K/Akt pathway (Meng et al., 2009). Furthermore, inhibition of MEK1/2 using PD98059 also reduced cell migration and invasion (Meng et al., 2009). In agreement with these observations, our results indicate that A549 lung cancer cell migration was significantly reduced by PI3K and MEK1/2 inhibitors, respectively 
(Figure 6c). Hence, menin may inhibit A549 cell migration partly by repressing PTN and its receptor RPTP $\beta / \zeta$ expression, and ectopic menin expression reduced the active and phosphorylated forms of FAK, PI3K and ERK1/2, but did not affect $\beta$-catenin expression and nuclear localization. However, the precise mechanism for downregulation of FAK, PI3K and ERK $1 / 2$ by menin remains to be analyzed.

Collectively, our findings unravel a previously unrecognized pathway in controlling A549 lung cancer cell migration, that is, the menin-PTN pathway. This mechanism is quite distinct from menin-regulated intercellular adhesion of pancreatic $\beta$-cells, in which menin interacts with the IQ motif containing GTPase activating protein 1, a scaffold protein, and reduces GTP-Rac1 interaction with IQ motif containing GTPase activating protein 1 , but increases E-cadherin/ $\beta$-catenin interaction with IQ motif containing GTPase activating protein 1 (Yan et al., 2009). Consistent with these findings, PTN is abundantly expressed in fetal lung epithelial cells of rats and enhances cell proliferation (Weng et al., 2009). Although PTN increases $\beta$-catenin phosphorylation and nuclear translocation in lung epithelial cells (Weng et al., 2009), we failed to observe an effect of PTN on $\beta$-catenin phosphorylation in A549 lung cancer cells (Supplementary Figure 6a). It is likely that the cancer cells respond distinctly to PTN stimulation. These findings suggest multiple roles for menin in regulating adhesion, migration and signal transduction in distinct types of cells.

Our results have provided new insight into meninregulated PTN/RPTP $\beta / \zeta$ signal transduction in controlling A549 lung cancer cell migration. These studies have unraveled the crucial mechanisms whereby menin represses migration at least partly through suppressing the PTN/RPTP $\beta / \zeta$ in repressing lung cancer migration. As menin actively represses PTN-induced lung cancer cell migration and loss of menin expression is closely correlated with enhanced lung cancer metastasis to lymph nodes, the menin-PTN pathway in regulating cell migration and metastasis may serve as a target for therapy against lung adenocarcinoma.

\section{Materials and methods}

Cell culture and gene transfection

MEF cell line generation and culture were previously described (Schnepp et al., 2006). A549 and NCI-H157 cell culture and polyethylenimine-mediated plasmid transfection were performed, as previously described (Gao et al., 2009). A549 cells were treated with or without recombinant human PTN (R\&D, Minneapolis, MN, USA), PI3K inhibitor (LY294002) (Cell Signaling, Danvers, MA, USA), and MEK1/2 inhibitor (U0126) (Cell Signaling).

\section{Western blotting}

The cells were lysed in radioimmunoprecipitation assay buffer (Jin et al., 2007), and the extracted proteins were resolved by sodium dodecyl sulfate-polyacrylamide gel electrophoresis before transfer onto PVDF membrane, and each of the antibodies was incubated against one of the following proteins: menin (Bethyl, Montgomery, AL, USA), PTN (Abnova, Walnut, CA, USA), RPTP $\beta / \zeta$ (BD, San Jose, CA, USA), FAK (Santa Cruz, Santa Cruz, CA, USA), Tyr397 phosphoFAK (Invitrogen, Carlsbad, CA, USA), ERK1/2 (Cell Signaling), Thr202/Tyr204 phospho-ERK1/2 (Cell Signaling), integrin $\alpha_{\mathrm{v}}$ (Millipore, Billerica, MA, USA), integrin $\beta_{3}$ (Santa Cruz), PI3K p85 (Cell Signaling), Tyr458 phospho-PI3K (Cell Signaling), $\beta$-catenin (Cell Signaling), Tyr142 phospho- $\beta$ catenin (Abcam, Cambridge, MA, USA) and $\beta$-actin (Santa Cruz). Membranes were further washed and incubated with an anti-rabbit

or

anti-mouse secondary antibody (Pierce, Rockford, IL, USA). Detection of immunoreactive bands was performed using an ECL detection kit (Pierce), according to the manufacturer's instructions. Equal protein loading was indicated by Ponceau-S staining of blotted membranes.

\section{IP assay}

IP assays were performed essentially, as previously described (Yan et al., 2009). Briefly, the harvested cells were lysed and incubated with a primary antibody or normal rabbit IgG (Millipore). Protein A or protein G-agarose beads (Santa Cruz) were added. Bound proteins were collected by centrifugation, washed and analyzed by western blotting.

\section{Cell migration assay and scratch wounding assay}

Migration assays were performed, as previously described (Mikelis et al., 2009), in 24-well transwell plates (Millipore) using uncoated polycarbonate membranes with $8 \mu \mathrm{m}$ pores. Serum-starved cells were harvested and re-suspended at a concentration of $1 \times 10^{4}$ cells per $0.2 \mathrm{ml}$ in serum-free medium containing $10 \%$ bovine serum albumin, and added to the upper chamber. At $24 \mathrm{~h}$ after incubation, non-migrated cells were scraped off the upper side of the filter, and filters were stained with $0.1 \%$ crystal violet. The number of migrated cells on the reverse side of the membrane was quantified by average cell counts from six random fields in each well, under a microscope at $\times 100$ magnifications (Supplementary Figure 1a). Each condition was assayed in triplicate wells, and the resulting data were subjected to statistical analysis using the Student's $t$-test. For scratch wound assay, subconfluent cells were scraped using sterilizing $10 \mu \mathrm{l}$ pipette tips, washed with phosphate-buffered saline and cultured in normal medium. The cells were observed under a Nikon Eclipse TE 300 inverted microscope (Nikon Corporation, Tokyo, Japan), and images were captured daily.

\section{Statistical analysis}

Values were presented as the mean \pm s.d. Statistical comparisons between groups were conducted using the Student's $t$-test, and $P<0.05$ was considered statistically significant.

\section{Conflict of interest}

The authors declare no conflict of interest.

\section{Acknowledgements}

This work is supported by an NFSC grant (No. 30701003, GH Jin) and a National Cancer Institute grant (R01CA113962, $\mathrm{XH})$. We appreciate the valuable comments from other members of our laboratories. 


\section{References}

Baillat G, Siret C, Delamarre E, Luis J. (2008). Early adhesion induces interaction of FAK and Fyn in lipid domains and activates raftdependent Akt signaling in SW480 colon cancer cells. Biochim Biophys Acta 1783: 2323-2331.

Carelli S, Zadra G, Vaira V, Falleni M, Bottiglieri L, Nosotti M et al. (2006). Up-regulation of focal adhesion kinase in non-small cell lung cancer. Lung Cancer 53: 263-271.

Chandrasekharappa SC, Guru SC, Manickam P, Olufemi SE, Collins FS, Emmert-Buck MR et al. (1997). Positional cloning of the gene for multiple endocrine neoplasia-type 1. Science $\mathbf{2 7 6}$ 404-407.

Chen JS, Wang Q, Fu XH, Huang XH, Chen XL, Cao LQ et al. (2009a). Involvement of PI3K/PTEN/AKT/mTOR pathway in invasion and metastasis in hepatocellular carcinoma: association with MMP-9. Hepatol Res 39: 177-186.

Chen YJ, Wei YY, Chen HT, Fong YC, Hsu CJ, Tsai CH et al. (2009b). Osteopontin increases migration and MMP-9 up-regulation via alphavbeta3 integrin, FAK, ERK, and NF-kappaBdependent pathway in human chondrosarcoma cells. $J$ Cell Physiol 221: $98-108$.

Debelenko LV, Brambilla E, Agarwal SK, Swalwell JI, Kester MB, Lubensky IA et al. (1997). Identification of MEN1 gene mutations in sporadic carcinoid tumors of the lung. Hum Mol Genet 6: 2285-2290

Debelenko LV, Swalwell JI, Kelley MJ, Brambilla E, Manickam P, Baibakov G et al. (2000). MEN1 gene mutation analysis of highgrade neuroendocrine lung carcinoma. Genes Chromosomes Cancer 28: 58-65.

del Pozo MA, Alderson NB, Kiosses WB, Chiang HH, Anderson RG, Schwartz MA. (2004). Integrins regulate Rac targeting by internalization of membrane domains. Science 303: 839-842.

Duces A, Karaky R, Martel-Renoir D, Mir L, Hamma-Kourbali Y, Bieche I et al. (2008). 16-kDa fragment of pleiotrophin acts on endothelial and breast tumor cells and inhibits tumor development. Mol Cancer Ther 7: 2817-2827.

Gao SB, Feng ZJ, Xu B, Wu Y, Yin P, Yang Y et al. (2009) Suppression of lung adenocarcinoma through menin and polycomb gene-mediated repression of growth factor pleiotrophin. Oncogene 28: $4095-4104$

Gao SB, Hua X, Jin GH. (2008). Menin regulates endocrine diseases by controlling histone modification and gene transcription. Ann Endocrinol (Paris) 69: 426-432.

Hall A. (1998). Rho GTPases and the actin cytoskeleton. Science 279: 509-514.

Hood JD, Cheresh DA. (2002). Role of integrins in cell invasion and migration. Nat Rev Cancer 2: 91-100.

Jager R, List B, Knabbe C, Souttou B, Raulais D, Zeiler T et al. (2002). Serum levels of the angiogenic factor pleiotrophin in relation to disease stage in lung cancer patients. Br J Cancer 86: 858-863.

Jin GH, Ma DY, Wu N, Marikar FM, Jin SZ, Jiang WW et al. (2007). Combination of human plasminogen kringle 5 with ionizing radiation significantly enhances the efficacy of antitumor effect. Int $J$ Cancer 121: 2539-2546.

Karnik SK, Hughes CM, Gu X, Rozenblatt-Rosen O, McLean GW, Xiong Y et al. (2005). Menin regulates pancreatic islet growth by promoting histone methylation and expression of genes encoding p27Kip1 and p18INK4c. Proc Natl Acad Sci USA 102: $14659-14664$.

Klein CA. (2008). Cancer. The metastasis cascade. Science 321: $1785-1787$.

Lemos MC, Thakker RV. (2008). Multiple endocrine neoplasia type 1 (MEN1): analysis of 1336 mutations reported in the first decade following identification of the gene. Hum Mutat 29: $22-32$.

Liu G, Meng X, Jin Y, Bai J, Zhao Y, Cui X et al. (2008). Inhibitory role of focal adhesion kinase on anoikis in the lung cancer cell A549. Cell Biol Int 32: 663-670.
Lu KV, Jong KA, Kim GY, Singh J, Dia EQ, Yoshimoto K et al. (2005). Differential induction of glioblastoma migration and growth by two forms of pleiotrophin. $J$ Biol Chem 280: 26953-26964.

McLean GW, Carragher NO, Avizienyte E, Evans J, Brunton VG, Frame MC. (2005). The role of focal-adhesion kinase in cancer-a new therapeutic opportunity. Nat Rev Cancer 5: 505-515.

Meng K, Rodriguez-Pena A, Dimitrov T, Chen W, Yamin M, Noda M et al. (2000). Pleiotrophin signals increased tyrosine phosphorylation of beta beta-catenin through inactivation of the intrinsic catalytic activity of the receptor-type protein tyrosine phosphatase beta/zeta. Proc Natl Acad Sci USA 97: 2603-2608.

Meng XN, Jin Y, Yu Y, Bai J, Liu GY, Zhu J et al. (2009). Characterisation of fibronectin-mediated FAK signalling pathways in lung cancer cell migration and invasion. Br J Cancer 101: 327-334.

Mikelis C, Sfaelou E, Koutsioumpa M, Kieffer N, Papadimitriou E. (2009). Integrin alpha(v)beta(3) is a pleiotrophin receptor required for pleiotrophin-induced endothelial cell migration through receptor protein tyrosine phosphatase beta/zeta. FASEB $J$ 23: 1459-1469.

Milne TA, Briggs SD, Brock HW, Martin ME, Gibbs D, Allis CD et al. (2002). MLL targets SET domain methyltransferase activity to Hox gene promoters. Mol Cell 10: 1107-1117.

Milne TA, Hughes CM, Lloyd R, Yang Z, Rozenblatt-Rosen O, Dou $Y$ et al. (2005). Menin and MLL cooperatively regulate expression of cyclin-dependent kinase inhibitors. Proc Natl Acad Sci USA 102: 749-754.

Molloy T, van 't Veer LJ. (2008). Recent advances in metastasis research. Curr Opin Genet Dev 18: 35-41.

Nelson WJ, Nusse R. (2004). Convergence of Wnt, beta-catenin, and cadherin pathways. Science 303: 1483-1487.

Palazzo AF, Eng CH, Schlaepfer DD, Marcantonio EE, Gundersen GG. (2004). Localized stabilization of microtubules by integrin- and FAK-facilitated Rho signaling. Science 303: 836-839.

Pannett AA, Thakker RV. (1999). Multiple endocrine neoplasia type 1. Endocr Relat Cancer 6: 449-473.

Pariser H, Ezquerra L, Herradon G, Perez-Pinera P, Deuel TF. (2005). Fyn is a downstream target of the pleiotrophin/receptor protein tyrosine phosphatase beta/zeta-signaling pathway: regulation of tyrosine phosphorylation of Fyn by pleiotrophin. Biochem Biophys Res Commun 332: 664-669.

Pei XH, Bai F, Smith MD, Xiong Y. (2007). p18Ink4c collaborates with Men1 to constrain lung stem cell expansion and suppress nonsmall-cell lung cancers. Cancer Res 67: 3162-3170.

Perez-Pinera P, Chang Y, Deuel TF. (2007). Pleiotrophin, a multifunctional tumor promoter through induction of tumor angiogenesis, remodeling of the tumor microenvironment, and activation of stromal fibroblasts. Cell Cycle 6: 2877-2883.

Petzmann S, Ullmann R, Klemen H, Renner H, Popper HH. (2001). Loss of heterozygosity on chromosome arm 11q in lung carcinoids. Hum Pathol 32: 333-338.

Polykratis A, Katsoris P, Courty J, Papadimitriou E. (2005). Characterization of heparin affin regulatory peptide signaling in human endothelial cells. $J$ Biol Chem 280: 22454-22461.

Schnepp RW, Chen YX, Wang H, Cash T, Silva A, Diehl JA et al. (2006). Mutation of tumor suppressor gene Men1 acutely enhances proliferation of pancreatic islet cells. Cancer Res 66: $5707-5715$.

Stoica GE, Kuo A, Powers C, Bowden ET, Sale EB, Riegel AT et al. (2002). Midkine binds to anaplastic lymphoma kinase (ALK) and acts as a growth factor for different cell types. J Biol Chem 277: 35990-35998.

Tamura M, Gu J, Tran H, Yamada KM. (1999). PTEN gene and integrin signaling in cancer. $J$ Natl Cancer Inst 91: 1820-1828.

von Wichert G, Jiang G, Kostic A, De Vos K, Sap J, Sheetz MP. (2003). RPTP-alpha acts as a transducer of mechanical force 
on alphav/beta3-integrin-cytoskeleton linkages. J Cell Biol 161: $143-153$.

Weng T, Gao L, Bhaskaran M, Guo Y, Gou D, Narayanaperumal J et al. (2009). Pleiotrophin regulates lung epithelial cell proliferation and differentiation during fetal lung development via beta-catenin and Dlk1. J Biol Chem 284: 28021-28032.

$\mathrm{Wu}$ X, Hua X. (2008). Menin, histone h3 methyltransferases, and regulation of cell proliferation: current knowledge and perspective. Curr Mol Med 8: 805-815.
Yan J, Yang Y, Zhang H, King C, Kan HM, Cai Y et al. (2009). Menin interacts with IQGAP1 to enhance intercellular adhesion of beta-cells. Oncogene 28: 973-982.

\section{This work is licensed under the Creative Commons} Attribution-NonCommercial-No Derivative Works
3.0 Unported License. To view a copy of this license, visit http://creativecommons.org/licenses/by-nc-nd/3.0/

Supplementary Information accompanies the paper on the Oncogene website (http://www.nature.com/onc) 